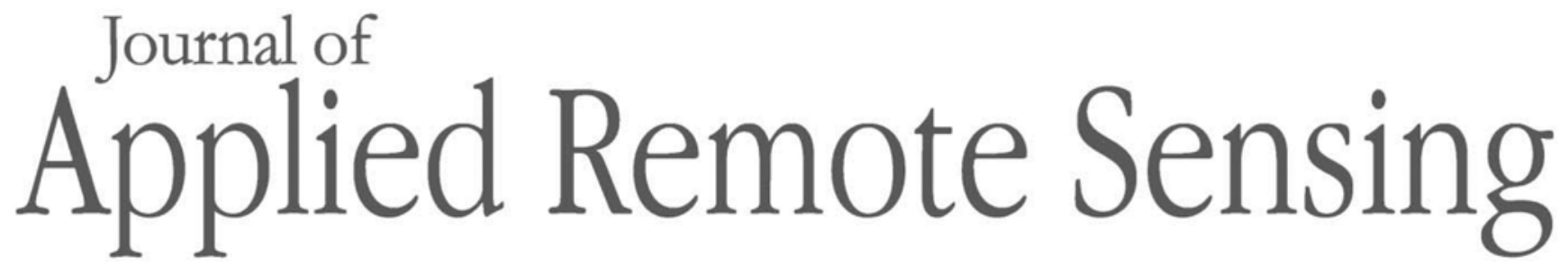

RemoteSensing.SPIEDigitalLibrary.org

\title{
Planning lunar observations for satellite missions in low-Earth orbit
}

\author{
Truman Wilson \\ Xiaoxiong Xiong
}




\title{
Planning lunar observations for satellite missions in low-Earth orbit
}

\author{
Truman Wilson ${ }^{\mathrm{a}, *}$ and Xiaoxiong Xiong ${ }^{\mathrm{b}}$ \\ ${ }^{a}$ Science Systems and Applications Inc., Lanham, Maryland, United States \\ ${ }^{\mathrm{b}}$ Sciences and Exploration Directorate, NASA/GSFC, Greenbelt, Maryland, United States
}

\begin{abstract}
For Earth-observing satellites in low-Earth orbit, radiometric calibration of the sensors on-orbit is critical for maintaining consistent Earth-view (EV) retrievals as the mission progresses. Many of these satellite instruments use on-board calibration targets, EV sites, and observations of celestial targets in order to perform the sensor characterization. Among the celestial targets, the Moon is widely used across a range of Earth-observing instruments in order to perform radiometric calibration, spatial characterization, and sensor intercomparison. Since many of these instruments use satellite maneuvers in order to bring the Moon into view at a desired time, calculating the time and geometric parameters of the observations is vital for mission planning purposes. We develop a simple tool for planning such observations of the Moon and other celestial targets for instruments in low-Earth orbit based on the SPICE toolkit developed by the Navigation and Ancillary Information Facility at NASA. Given a set of satellite orbital data along with a definition of the instrument coordinates, the tool is designed to provide the timing of observations for an arbitrary view-port direction and a maneuver along an arbitrary axis relative to the spacecraft. The tool can be tested versus known lunar observations for the Aqua and Terra moderate resolution imaging spectroradiometer and the Suomi-NPP and NOAA-20 visible infrared imaging radiometer suite instruments for both roll and pitch maneuvers. We also perform simulations of lunar observations for different instrument configurations, orbits, and maneuver types in order to analyze the change in the potential lunar observations. Finally, we show a simple extension of the tool which can be used for identifying planet and star observations. (C) 2019 Society of Photo-Optical Instrumentation Engineers (SPIE) [DOI: 10.1117/1.JRS .13.024513]
\end{abstract}

Keywords: moderate resolution imaging spectroradiometer; visible infrared imaging radiometer suite; lunar calibration; satellite maneuvers; reflective solar bands.

Paper 190059 received Jan. 25, 2019; accepted for publication Apr. 16, 2019; published online May 9, 2019.

\section{Introduction}

Calibration and characterization of Earth-observing instruments on-orbit have been a critical component to the success of many satellite-based climate and weather monitoring missions. Instruments such as the moderate resolution imaging spectroradiometer (MODIS) on board the Terra and Aqua spacecrafts and the visible infrared imaging radiometer suite (VIIRS) on board the Suomi-NPP (SNPP) and NOAA-20 (N20) spacecrafts use a variety of methods for sensor calibration and characterization. ${ }^{1,2}$ The primary method uses sets of on-board calibrators (OBC) such as a solar diffuser (SD) and its associated SD stability monitor for calibrating the reflective solar bands (RSB) and a blackbody source for calibrating the thermal emissive bands (TEB). In addition to the OBC, external calibration sources have also been used for sensor characterization, which includes the Moon, ${ }^{3,4}$ Earth-view (EV) targets, ${ }^{5,6}$ and stars. ${ }^{7}$ The Moon in particular has a long history of use as a calibration source for both MODIS and VIIRS due to its stable, well-characterized radiometric behavior. ${ }^{8}$ It has been used in RSB calibration for a number of other instruments, including SeaWiFS, ${ }^{9}$ MISR, ${ }^{10}$ and the Landsat Operational Land Imager (OLI), ${ }^{11}$ among others.

*Address all correspondence to Truman Wilson, E-mail: truman.wilson@ssaihq.com

$1931-3195 / 2019 / \$ 25.00$ (C) 2019 SPIE 
For MODIS and VIIRS, spacecraft roll maneuvers are performed in order to make lunar observations on a near monthly basis. In order to increase the calibration consistency between lunar observations, the lunar phase angle (the angle between the Sun, Moon, and instrument) $\alpha_{\text {SMI }}$ is typically restricted to be within a 1-deg range, which reduces the change in the geometric correction factors derived from the USGS ROLO model that need to be applied to each observation when using the Moon for radiometric calibration. ${ }^{8}$ For MODIS, previous work showed that observations with high-phase angle differences (up to $30 \mathrm{deg}$ ) can show differences in the measured gain of up to $4 \%$ for some spectral bands. ${ }^{12}$ The Moon is viewed through the instrument space-view (SV) port, and a roll maneuver is required in order to align this port with the Moon when it is within the desired phase angle range. This means that calculating the precise timing and geometry of candidate observations including potential restrictions to the spacecraft's maneuvering ability will be necessary in order to ensure that the Moon is viewed under the desired conditions.

In the previous work, tools were developed which were able to accurately predict the observation parameters for the lunar roll maneuvers used for MODIS and VIIRS. ${ }^{13-15}$ However, we wished to extend these tools in order to generalize the calculations for an arbitrary instrument viewport, arbitrary axis of rotation, and an arbitrary target. These capabilities have several potential impacts. One of the main advantages is that it provides the capability for computing the timing and angular offset of the Moon for pitch maneuvers using the same tool, which not only uses a different rotation axis, but also views the Moon through the instrument EV port instead of the SV port. Since the EV port has a wide field of view (FOV) compared to the $\mathrm{SV}$, we can also choose an arbitrary offset within this port in order to view the Moon at a specific angle relative to the instrument optics. It also allows us to change our target from the Moon to other celestial objects, such as bright stars and planets, which can be observed by the VIIRS day/night band (DNB). ${ }^{7}$ Accurate predictions of these observation times will allow us to go back through the historical data in order to extract useful data for calibration and sensor characterization. It will also allow for us to simulate observations under different instrument pointing and spacecraft orbital configurations, which can be helpful for planning changes to current or future missions. Although the analysis in this work focuses on whisk broom, scanning radiometers such as MODIS and VIIRS, the methodology we develop is independent of the type of imager since we only require knowledge of the relative geometry of the maneuvering and view-port axes. Therefore, push broom sensors, such as the OLI, ${ }^{11}$ MISR,,${ }^{10}$ and ASTER ${ }^{16}$ on the Terra platform, could also be used if desired.

To facilitate the development of this tool, we use the SPICE toolkit developed by NASA's Navigation and Ancillary Information Facility, ${ }^{17}$ which provides ephemeris data for the Sun, planets, and the Moon as well as providing tools to allow us to generate satellite orbital data and reference frames from input data. To find observation times, we use the Geometry Finder Tool, which is a set of algorithms in SPICE designed to find time ranges associated with input geometrical restrictions, such as phase angle restrictions, relative vector offsets, occultation, and illumination angles, among others. These algorithms can be applied to efficiently narrow down the time ranges associated with the observation until an optimum event time is found. Using the event timing, we can calculate the parameters needed for our calibration and characterization activities, such as the phase angle, lunar libration angles, and oversampling factor as used in the case of our routine lunar calibration. ${ }^{3}$ In principle, the methodology developed in this work can be implemented in other, more generalized mission planning tools if desired, such as the General Mission Analysis Tool, or GMAT, developed by a collaboration between NASA, private industry, and other individual contributors. ${ }^{18}$

In this work, we will discuss the development of our scheduling tool starting with the coordinate system definitions in Sec. 2. We will then compare our predictions using this tool to our past lunar observations from roll maneuvers and pitch maneuvers in Sec. 3 and 4, respectively. In Sec. 3.1, we will show the change in possible lunar observations using different SV port locations and for orbits at different equatorial crossing times, using SNPP orbital data as a base for these simulations. In Sec. 4.1, we will analyze pitch observation opportunities and use simulated orbital data to find several candidate observations for future milestone years for the MODIS and VIIRS instruments. We will then show an extension of the planning tool for finding planetary 
and stellar observation opportunities in Sec. 5. Finally, in Sec. 6, we will present our summary and conclusions.

\section{Definition of the Instrument and Observation Coordinate Systems}

In order to determine the timing of potential satellite observations, we first need to properly define the relevant coordinate systems. For most instruments on board Earth-observing satellites, the EV observation port is pointed at nadir in a geocentric configuration for data acquisition. Therefore, it is convenient to define the following coordinate system, which we will call the instrument coordinate system (ICS). In this coordinate system, the $z$ axis is defined to be pointing from the spacecraft toward the center of the Earth. The $x$ axis is perpendicular to $z$ and is in the instrument orbital plane pointing in the direction of orbital motion. The $y$ axis is normal to the orbital plane and can be found from the cross product of the $z$ and $x$ axes. These axes can be computed from the instrument position and velocity vectors, $\vec{r}_{\text {sat }}$ and $\vec{v}_{\text {sat }}$, which are computed from instrument orbital data in the Earth-centered inertial (ECI) J2000 reference frame. A diagram of the ICS is shown in Fig. 1(a) and can be summarized by the following set of equations:

$$
\begin{aligned}
& \hat{x}=\frac{-\vec{r}_{\text {sat }} \times\left(\vec{r}_{\text {sat }} \times \vec{v}_{\text {sat }}\right)}{\left|\vec{r}_{\text {sat }} \times\left(\vec{r}_{\text {sat }} \times \vec{v}_{\text {sat }}\right)\right|} \\
& \hat{y}=\hat{z} \times \hat{x} \\
& \hat{z}=-\frac{\vec{r}_{\text {sat }}}{\left|\vec{r}_{\text {sat }}\right|} .
\end{aligned}
$$

We can create the ICS in SPICE by generating a reference frame kernel from the input satellite orbital data. ${ }^{17}$ This can be done in either a geocentric configuration as defined by Eq. (1), or in a geodetic configuration, where the ICS $z$ axis points perpendicular to the ellipsoidal surface of the Earth, as is the case for the VIIRS instruments. ${ }^{19}$ However, for observations that use spacecraft maneuvers, the ICS is not ideal since the rotation axis and view axis could be pointing in an arbitrary direction. Instead, we will define a new coordinate system with a fixed offset relative to the ICS which we will call the observation coordinate system (OCS). The OCS will be defined by the principle axes $\hat{i}, \hat{j}$, and $\hat{k}$. In the OCS, the $k$ axis is aligned with the desired axis of rotation for the maneuver, as seen in Fig. 1(b). The OCS is further rotated such that the viewport is in the $i k$ plane. We can describe the pointing direction of the viewport in the OCS in latitudinal coordinates. Using this formulation, the viewport latitude $V_{\text {lat }}$ represents a cone (or plane if $V_{\text {lat }}=0$ ) of observation, in which the viewport is rotated. When the $\operatorname{target}$ latitude $T_{\text {lat }}$ is equal to $V_{\text {lat }}$ in the OCS, then an observation is possible through the viewport if a rotation is performed, as seen in
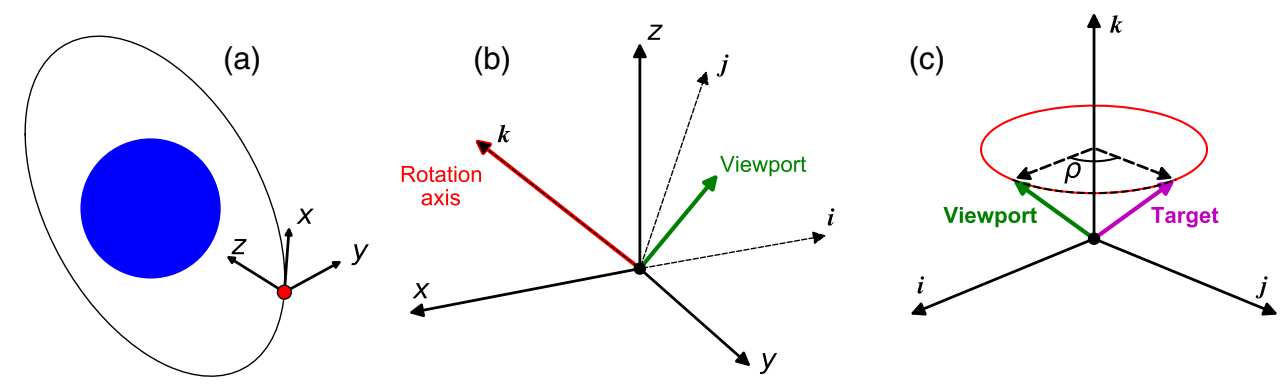

Fig. 1 Diagram of the coordinate systems used for observation scheduling. (a) The definition of the instrument coordinate system or ICS. (b) The transformation of the ICS, represented by $x, y$, and $z$ to the observation coordinate system, or OCS, represented by $i, j$, and $k$. The $k$ axis is aligned with the rotation axis for the instrument maneuver. The $i$ axis is aligned such that the instrument viewport is in the $i k$ plane. (c) The observation geometry in the OCS. The viewport and target vectors are labeled and shown in green and purple, respectively. The viewport path during a rotation is shown as a red circle, and the angle between the viewport and target along this path is denoted by $\rho$. 
Fig. 1(c). In the OCS, the target longitude $T_{\text {lon }}$ represents the angular offset of the target. The instrument requires a rotation of angle $\rho=T_{\text {lon }}$ in order to observe the object through the viewport. These general criteria for observations are listed in

$$
\begin{aligned}
& T_{\text {lat }}=V_{\text {lat }} \\
& \rho_{\text {min }} \leq T_{\text {lon }} \leq \rho_{\text {max }},
\end{aligned}
$$

where $\rho_{\min }$ and $\rho_{\max }$ define the allowed maneuver range.

To test the accuracy of the tool, we compared our observation predictions to each of the past MODIS and VIIRS lunar roll maneuvers ${ }^{3,4}$ in Sec. 3 and pitch maneuvers ${ }^{20-22}$ in Sec. 4. Although these events have been scheduled in the past using other tools, ${ }^{13,15}$ they provide a set of test data which will give us confidence in the results of our simulations which will be presented in Secs. 3.1 and 4.1. Since 2017, the MODIS and VIIRS Characterization and Support Teams (MCST/VCST) have used this version of the tool along with the previous tools for planning and verification of the MODIS and VIIRS lunar observation parameters.

\section{Lunar Observations from Roll Maneuvers}

The most common type of observation we are interested in for our work with MODIS and VIIRS is the regularly scheduled lunar observation using a roll maneuver (about the $x$ axis in the ICS). ${ }^{3,4}$ The Terra, Aqua, SNPP, and N20 satellites are each in sun-synchronous orbits. For Terra, the orbit is descending (north to south) during satellite daytime with an equatorial crossing time of 10:30 local time. For Aqua, SNPP, and N20, the orbit is ascending (south to north) during satellite daytime with equatorial crossing times at $\sim 13: 30$ local time. The relative orbits of each of the instruments are shown in Fig. 2(a).

For both instruments, lunar observations are used primarily for RSB calibration and occur on a near-monthly basis. In the case of MODIS, the observations occur at a different angle of incidence (AOI) on the scan mirror than the SD, which allows us to derive the scan mirror responseversus-scan-angle, or RVS. ${ }^{23}$ The calibration look-up tables are also generated on a near-monthly basis coinciding with these observations, or when instrument gain changes measured using SD or EV data are observed, in order to maintain the highest possible data quality. For the MODIS $\mathrm{RSB}$, the top-of-atmosphere reflectance has a total uncertainty requirement of $2 \%$. There is no additional requirement given to the individual contributors to this total uncertainty, which includes the uncertainty from the lunar observations. ${ }^{24}$ The lunar uncertainty is derived using the standard deviation of the fitting residuals of an empirical piece-wise fit to the time-series data, which has peak-to-peak differences on the order of $1 \%$ or less owing to the libration effect. ${ }^{3,8,24}$ Near-monthly observations of the Moon are helpful for fitting the piecewise function appropriately through these libration oscillations. The relative uncertainty for MODIS lunar calibration for the RSB is averaged annually and is on the order $0.2 \%$ to $0.3 \%$. Absolute uncertainties associated with the geometric corrections derived from the

(a)

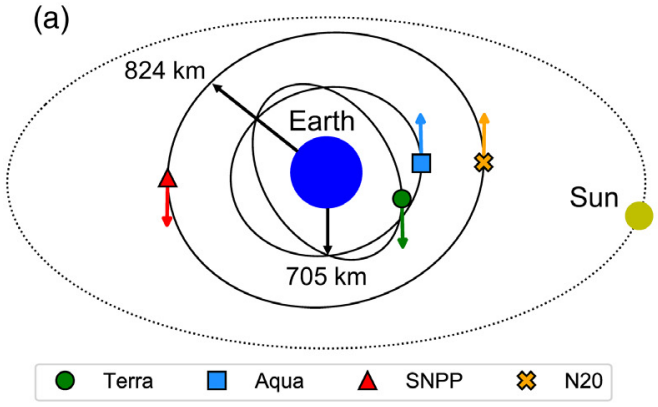

(b)

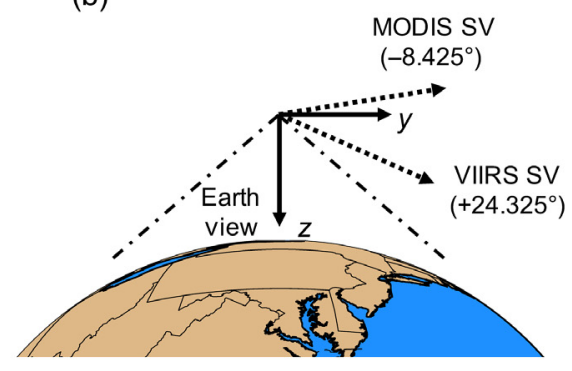

Fig. 2 (a) A diagram of the relative orbits of the Terra, Aqua, SNPP, and N20 satellites (not to scale) and (b) a diagram showing the relative orientation of the EV and SV ports for both MODIS and VIIRS. 
ROLO model are on the order of $5 \% .{ }^{8}$ However, the lunar calibration results are normalized to the derived prelaunch gain values measured at the SV AOI on the scan mirror.

For VIIRS, the AOI of the lunar observations is at the same AOI as that of the SD. Therefore, the RVS for VIIRS is not derived directly using lunar observations. In this case, the lunar data are used to provide small corrections to the SD degradation data in order to match the trending SD $F$ factor (inverse of the gain) with the derived lunar $F$ factor. ${ }^{4}$ These updates using lunar data are only applied periodically throughout the mission, but do require frequent observations in order to account for lunar librations as was the case for MODIS. The VIIRS RSB also has an uncertainty requirement of $2 \%$ in the top-of-atmosphere reflectance. ${ }^{25}$ For the VIIRS lunar data, an empirical fit is applied to reduce the libration oscillations, resulting in a relative accuracy of around $0.1 \%$.

For each instrument, the Moon is viewed through the SV port, which is located in the $y z$ plane of the ICS at $-8.425 \mathrm{deg}$ and $+24.325 \mathrm{deg}$ away from the $y$ axis toward the $z$ axis for MODIS and VIIRS, respectively, as shown in Fig. 2(b). For VIIRS, the SV is an extension of the EV port, whereas MODIS has a separate SV port. During the lunar data collect, the EV data collection sector is realigned to include the $\mathrm{SV}$, ensuring that the entire range of the SV port is observed. For VIIRS, the EV sector pixels use subpixel aggregation in order to have better consistency in the along-scan ground resolution over the full range of view angles. From nadir to $31.7 \mathrm{deg}$, three subpixels are averaged per EV pixel (aggregation zone 3), from $31.7 \mathrm{deg}$ to 44.9 deg 2 subpixels are averaged per EV pixel (aggregation zone 2), and above $44.9 \mathrm{deg}$ only 1 pixel is used (aggregation zone 1). For SNPP, the EV sector realignment centers the $\mathrm{SV}$ port in the sector, putting the lunar image in aggregation zone 3 , which has an equal aspect ratio in the scan and track directions owing to the subpixel averaging. For N20, the EV sector realignment puts the SV near the edge of scan in order to view more of the Earth during the scan. This puts the lunar image in aggregation zone 1, with a scan-to-track aspect ratio of $3: 1$. An example image of the Moon for each of the MODIS and VIIRS sensors can be seen in Fig. 3.

For MODIS and VIIRS, the lunar observation possibilities will be limited by the location of the SV port and the maneuvering range of the spacecraft. We define the lunar phase angle $\alpha_{\mathrm{SMI}}$ as the angle between the Sun, Moon, and instrument, as seen in Fig. 4. Although the full phase angle range observed can be as high as $50 \mathrm{deg}$ for both MODIS and VIIRS, for calibration purposes, this range is restricted to a selected 1-deg range for most observations for two reasons.
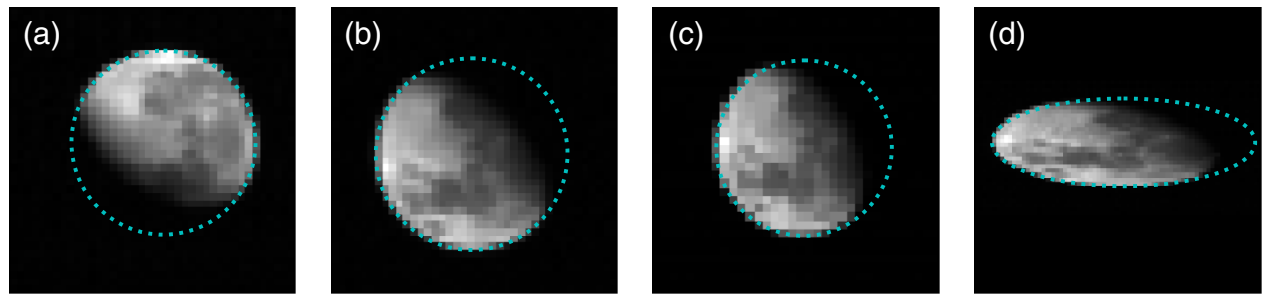

Fig. 3 Lunar images for Aqua and Terra MODIS and SNPP and N20 VIIRS from February of 2018. The blue dotted curves show the result of the edge fitting to locate the center position of the Moon. (a) Terra MODIS on February 4, 2018, (b) Aqua MODIS on February 25, 2018, (c) SNPP VIIRS on February 26, 2018, and (d) N20 VIIRS on February 26, 2018. For N20, the Moon is viewed in aggregation zone 1 (no subpixel aggregation), resulting in an elliptical image with an aspect ratio of $3: 1$.

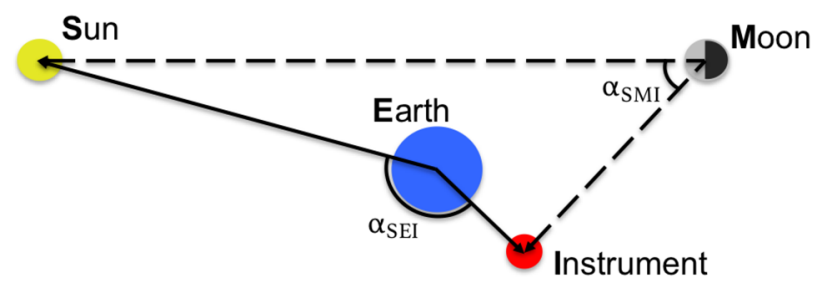

Fig. 4 A diagram of the relative positions of the Sun (S), Earth (E), Moon (M), and instrument (I) as it relates to the definition of the phase angles $\alpha_{\mathrm{SMI}}$ and $\alpha_{\mathrm{SEl}}$. 
Table 1 The scheduled lunar roll maneuver parameters for Aqua and Terra MODIS and SuomiNPP and NOAA-20 VIIRS. The error listed in the timing accuracy is the standard deviation of the difference for all of the events. The number of events is listed through January 1, 2019.

\begin{tabular}{lccccc}
\hline \hline Instrument & Launch year & Events & Phase $\left(\alpha_{\text {SMI }}\right)(\mathrm{deg})$ & Roll range $(\rho)(\mathrm{deg})$ & Timing accuracy $(\mathrm{s})$ \\
\hline Terra MODIS & 1999 & 186 & 55.0 to 56.0 & -20 to 0 & $0.21 \pm 0.44$ \\
Aqua MODIS & 2002 & 170 & -55.0 to 56.0 & -20 to 0 & $0.54 \pm 0.49$ \\
SNPP VIIRS & 2011 & 61 & -50.5 to 51.5 & -15 to 0 & $-0.24 \pm 0.59$ \\
N20 VIIRS & 2017 & 9 & -50.5 to 51.5 & -15 to 0 & $0.90 \pm 0.27$ \\
\hline \hline
\end{tabular}

First, a large phase angle range can result in biases in the calculated gain based on the geometric correction factors used to compare events. ${ }^{12}$ Second, the selected phase angle range provides the maximum number of observations throughout the year given the relative orbits, SV pointing direction, and spacecraft maneuvering range (see Sec. 3.1). The observation parameters can be seen in Table 1. For the values of $\alpha_{\mathrm{SMI}}$, we will use negative values to refer to the waxing phase of the Moon and positive values to refer to the waning phase of the Moon. For scheduling lunar observations, we apply the phase angle restriction first before applying the criteria from Eq. (2) in order to reduce the time range over which we are computing the observation geometries. The observation criteria for roll maneuver observations is thus

$$
\begin{aligned}
& \alpha_{\text {min }}<\alpha_{\text {SMI }}<\alpha_{\text {max }} \\
& T_{\text {lat }}=V_{\text {lat }} \\
& \rho_{\text {min }} \leq T_{\text {lon }} \leq \rho_{\text {max }} .
\end{aligned}
$$

Here $T_{\text {lat }}, T_{\text {lon }}$, and $V_{\text {lat }}$ are defined in the OCS as described previously. A diagram of the time range restrictions for each applied criterion can be seen in Fig. 5(a) for an example observation in Terra MODIS. The applied phase angle restriction is shown in Fig. 5(b) and the observation plane and maneuvering restrictions are shown in Fig. 5(c).
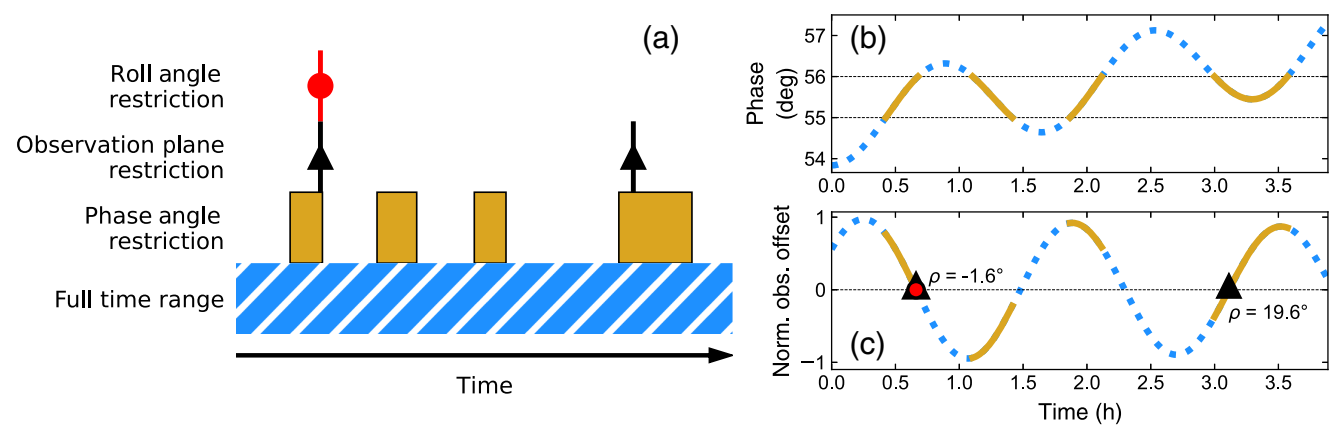

Fig. 5 Diagram of the time range restrictions applied for an example lunar roll maneuver for Terra MODIS. (a) An example of how the time range is restricted as each geometric criterion is applied to the orbital data from bottom to top. Symbols are added to the observation plane and roll angle restrictions since they occur at a single time. These restrictions are applied to the data in (b) and (c), with the colors, patterns, and symbols matching each time period. (b) The phase angle $\alpha_{\mathrm{SMI}}$ versus time. The thin dashed lines show the preferred phase angle range for Terra MODIS between $55 \mathrm{deg}$ and $56 \mathrm{deg}$. The blue dotted line shows the phase angle over the full-time period and the solid yellow lines show when the phase angle is within the desired range. (c) The normalized distance of the Moon to the observation plane versus time. The lines show this distance for the same time ranges and restrictions as (b), with the black triangles added to show when the Moon is in the observations plane. The red circle shows when the roll angle criterion is also met. The roll angles of each candidate event are listed on the plot. 
We can compare the calculated observation times to the actual observation times by determining when the Moon is centered in the FOV. Since both MODIS and VIIRS view a full disk image of the Moon over multiple scans, computing this time is simple. First, we determine the position of the Moon in each scan by fitting a circle (or ellipse) to an edge-filtered image of the Moon, as seen in Fig. 3. We can then interpolate the lunar position versus scan time to give us the time that the Moon is at the center of the FOV, which we then compare to the calculated observation time.

The timing accuracy for both MODIS and VIIRS instruments is listed in Table 1. In each case, the difference between our predicted and measured observation times are less than the time of a single scan, which is nominally $1.48 \mathrm{~s}$ for MODIS and $1.78 \mathrm{~s}$ for VIIRS. For VIIRS, the observation times were predicted using a geodetic pointing configuration as opposed to the geocentric configuration used for MODIS. ${ }^{19}$ Using a geocentric pointing configuration results in timing accuracies of $-0.46 \pm 2.26$ and $2.31 \pm 3.22 \mathrm{~s}$ for SNPP and N20 VIIRS, respectively. However, since the maneuvers are performed minutes before the observation and the Moon is visible for many scans, the pointing configuration makes little practical difference for scheduling maneuvers for lunar calibration of these instruments.

With the observation times, we can calculate the observation parameters, which include the phase angle, roll angle, oversampling factor, and libration angles, among many others. In Fig. 6, we show a comparison between the parameters for Aqua MODIS and SNPP VIIRS throughout each mission. Aqua and SNPP are in similar daytime ascending orbits, with equatorial crossing times at $\sim 13: 30$ local time. In Fig. 6(a), we can see that while most events fall within the phase angle ranges outlined in Table 1, there are several outlier events for each mission outside of this range. This occurs in months where the lunar orbit geometry prevents an observation at the desired phase angle with the given roll maneuver restrictions and another phase angle is chosen inside the allowed range. It should be noted that while the SNPP roll angle range goes out to $-15 \mathrm{deg}$; in practice, the magnitude of the largest roll angle used throughout the mission at this point has been less than $-11 \mathrm{deg}$. Also, some events occur outside of the specified roll angle ranges but do not perform maneuvers beyond those bounds. Instead, they rely on the extended FOV of the SV port in order to observe the Moon.

Oversampling is an important consideration for radiometric calibration using the Moon. Since sensors like MODIS and VIIRS are designed to have continuous images from scanto-scan at the EV nadir, when viewing the Moon at a much greater distance, there is significant scan-to-scan overlap in the images, causing oversampling. For MODIS and VIIRS, the
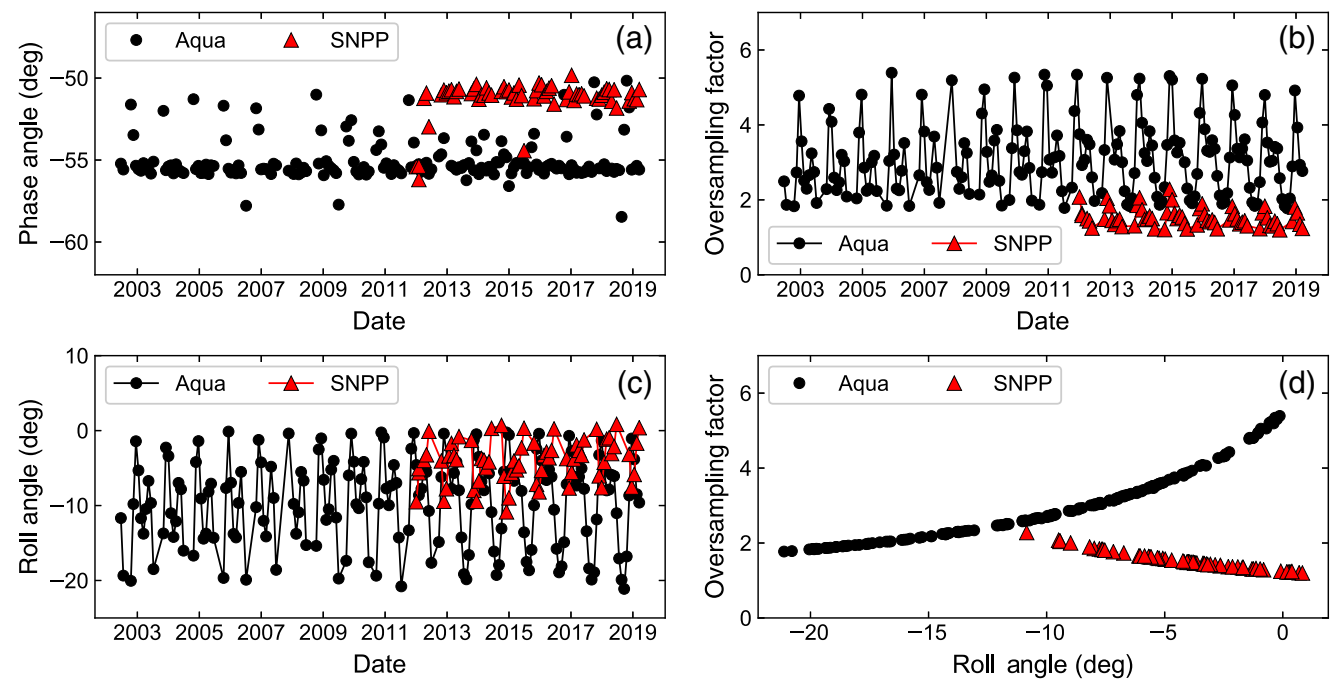

Fig. 6 Plots of selected parameters related to lunar roll maneuvers for Aqua MODIS (black circle) and SNPP VIIRS (red triangle): (a) the phase angle $\alpha_{\text {SMI }}$ for each event, (b) the oversampling factor for each event for the 1-km bands in MODIS and the 750-m bands in VIIRS, (c) the roll angle for each event, and (d) the correlation between the oversampling factor and the roll angle. In (b) and (c), lines are added to the plots in order to highlight the periodic nature of the parameter over time. 
oversampling effect allows for the construction of a lunar image for each individual detector within a given band. In order to calculate the radiance for the detector images, the integrated pixel-by-pixel radiance must be divided by the oversampling factor in order to get an accurate value for the lunar radiance. ${ }^{3}$ The oversampling factor $f_{\text {os }}$ can be described simply as the ratio of the pixel size on the lunar surface $P$, and the distance the pixel moves each scan $d$ as follows:

$$
\begin{aligned}
& f_{\text {os }}=\frac{P}{d} \\
& P=\frac{\left|\vec{r}_{I M}\right|}{h} R \\
& d=\left|\vec{v}_{t}\right| s .
\end{aligned}
$$

In the definition of the pixel size $P,\left|\vec{r}_{I M}\right|$ is the instrument-Moon distance, $h$ is the nominal instrument altitude, and $R$ is the EV pixel size at nadir. For the definition of the pixel distance per scan $d,\left|\vec{v}_{t}\right|$ is the Moon's speed relative to the satellite track direction and $s$ is the amount of time it takes to complete each scan. In Ref. $3, \vec{v}_{t}$ was derived in the ECI J2000 reference frame as

$$
\vec{v}_{t}=\vec{v}_{I}\left(1-\frac{\left|\vec{r}_{I M}\right|}{\left|\vec{r}_{I}\right|} \sin (\rho+\delta)-\frac{\vec{v}_{I} \cdot \vec{v}_{M}}{\left|\vec{v}_{I}\right|^{2}}\right),
$$

where $I$ and $M$ represent the instrument and Moon, respectively, $\rho$ is the roll angle, and $\delta$ is the SV port offset from the $y$ axis of the ICS. Since the direction of instrument motion for MODIS and VIIRS is the same as the track direction, $\vec{v}_{t}$ is proportional to $\vec{v}_{I}$, with the additional factor on the right side of Eq. (5) accounting for the spacecraft orbital motion (second term) and the relative motion of the spacecraft and Moon (third term). With the definition of the OCS, the motion of all objects is relative to the instrument and the track direction is along the $k$ axis. The Moon's velocity in the OCS can be computed easily using SPICE, and therefore, $\vec{v}_{t}$ can be written more simply as

$$
\vec{v}_{t}=\left(\vec{v}_{M, \mathrm{OCS}} \cdot \hat{k}\right) \hat{k}
$$

Using our definition of the OCS, the oversampling factor for the MODIS and VIIRS lunar observations can be written as

$$
f_{\mathrm{os}}=\frac{\left|\vec{r}_{M, \mathrm{OCS}}\right| R}{\left|\vec{v}_{M, \mathrm{OCS}} \cdot \hat{k}\right| h s} .
$$

We can note here that Eq. (7) does not use the parameters for the roll angle $\rho$ or the SV port offset $\delta$. These parameters are folded into the values of $\vec{r}_{M \text {,OCS }}$ and $\vec{v}_{M \text {,OCS }}$ when derived in the OCS.

Since the roll angle determines angular range that the viewport FOV traces out during the observation, the oversampling factor is correlated with the magnitude of the roll angle as seen in Fig. 6(d). The time series of the oversampling factor and roll angle for Aqua MODIS and SNPP VIIRS can be seen in Figs. 6(b) and 6(c), respectively. Since both instruments roll in the same direction but have view-ports pointing in opposite directions about the ICS $y$ axis [see Fig. 2(b)], the oversampling factor decreases with roll maneuvers for MODIS, whereas it increases for VIIRS.

Aside from the scheduled Moon observations, the tool can be used to predict the timing of lunar intrusions on the instrument SV ports at any value of $\alpha_{\mathrm{SMI}}$, otherwise, known as an unscheduled Moon observation. ${ }^{26,27}$ These observations are convenient because they do not require spacecraft maneuvers and they occur over many successive orbits. Although there are more of these observations than scheduled lunar observations, the difference in $\alpha_{\text {SMI }}$ between observations can be quite large and can cause a difference in results of the calibration of a few percent due to the changing geometry of the observation. ${ }^{12}$ However, they can be useful as an additional data set for other instrument characterizations, such as the spatial characterization and the electronic crosstalk correction. ${ }^{28}$ 


\subsection{Roll Maneuver Orbit Simulations}

Planning lunar observations has been a key component of the MODIS and VIIRS calibration activities throughout each mission. As future missions are planned and become operational, it will be important to know the possible geometries of lunar observations in a variety of configurations. These missions may need to use different maneuver types, have a different observation port configuration, or be in a different orbit than any current mission, which necessitates the ability to predict various types of lunar observations. In order to understand the observation geometries under a variety of conditions, we perform simulations of observations using orbital data from the SNPP satellite during the year 2017 as the baseline for our simulations. We can then add offsets to the instrument view port as well as alter the orbit in order to analyze the different types of observations that occur under these conditions. In principle, this simulation can be performed for an arbitrary year, however, it would only result in minor changes to the predicted observations, mainly owing to the precession of the lunar orbit tilt relative to the ecliptic. $^{15}$

The first simulation we perform is for observations using different viewports in the same plane as the VIIRS instrument (the $y z$ plane in the ICS). For this work, we will use the nominal viewport direction of the VIIRS instrument, the viewport direction of the MODIS instrument, and the opposite viewport direction of the VIIRS instrument at $+24.325 \mathrm{deg},-8.425 \mathrm{deg}$, and $-24.325 \mathrm{deg}$ from the $y$ axis of the ICS, respectively, as shown in Fig. 7(a). We can then calculate all of the possible observations using roll maneuvers (about the $x$ axis of the ICS) for our simulated year with a roll angle restriction between $-15 \mathrm{deg}$ and $0 \mathrm{deg}$ (the nominal roll angle range for the VIIRS instrument, see Table 1). The histograms in Fig. 7(b) show that for the $+24.325 \mathrm{deg}$ and $-8.425 \mathrm{deg}$ pointing configuration, the numbers of observations at each phase angle are approximately equal, whereas the $-24.325 \mathrm{deg}$ pointing configuration shows more observations at higher and lower phase angles. Although the $+24.325 \mathrm{deg}$ and -8.425 deg have opposite pointing directions, the FOV that each configuration traces out within the given maneuver range are similar when the orbital offset is accounted for. However, this also means that the observations of similar phase angles occur at opposite points in the orbit.

The second simulation we perform is for VIIRS-like instruments in orbits at different equatorial crossing times. The nominal crossing time is at 13:25 local time, which produces the same observations as the $+24.325 \mathrm{deg}$ viewport data in Fig. 7 . We also calculated the possible observations for a 15:25 and 17:25 equatorial crossing time as seen in Fig. 8, with the same maneuver range discussed previously. In Fig. 8(a), we show a diagram of the range of possible FOVs for each orbit, represented by concentric circles, with the outer circle representing the nominal pointing direction of the SV during the orbit, and the inner circle representing the pointing of the SV at the maximum roll angle of $-15 \mathrm{deg}$. The area in between the two circles represents the full range of potential SV FOVs. For the 15:25 orbit, both the 13:25 and 17:25 orbits have overlapping

(a)
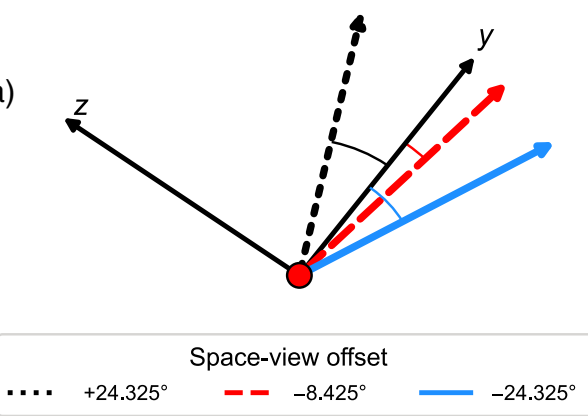

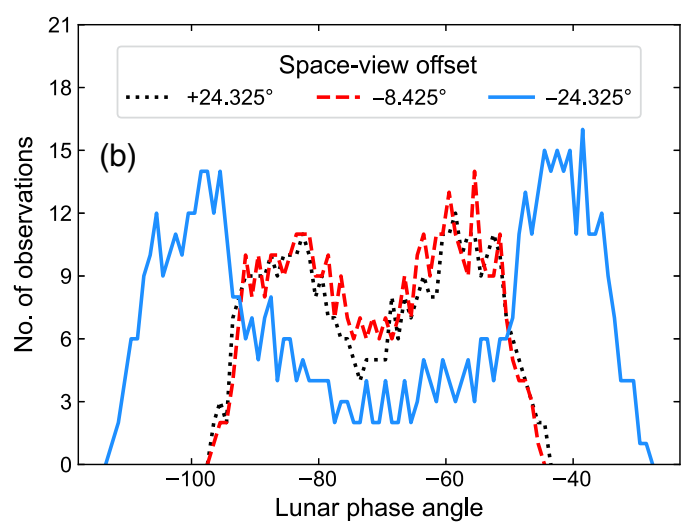

Fig. 7 (a) A diagram of the SV pointing geometry for three different configurations. In each case, the SV is chosen to be in the $y z$ plane of the ICS at the specified angular offsets from the $y$ axis. (b) Histograms of the number of observation opportunities at a given lunar phase angle in 1-deg bins for each pointing configuration in (a). These histograms show simulated data from 2017. 

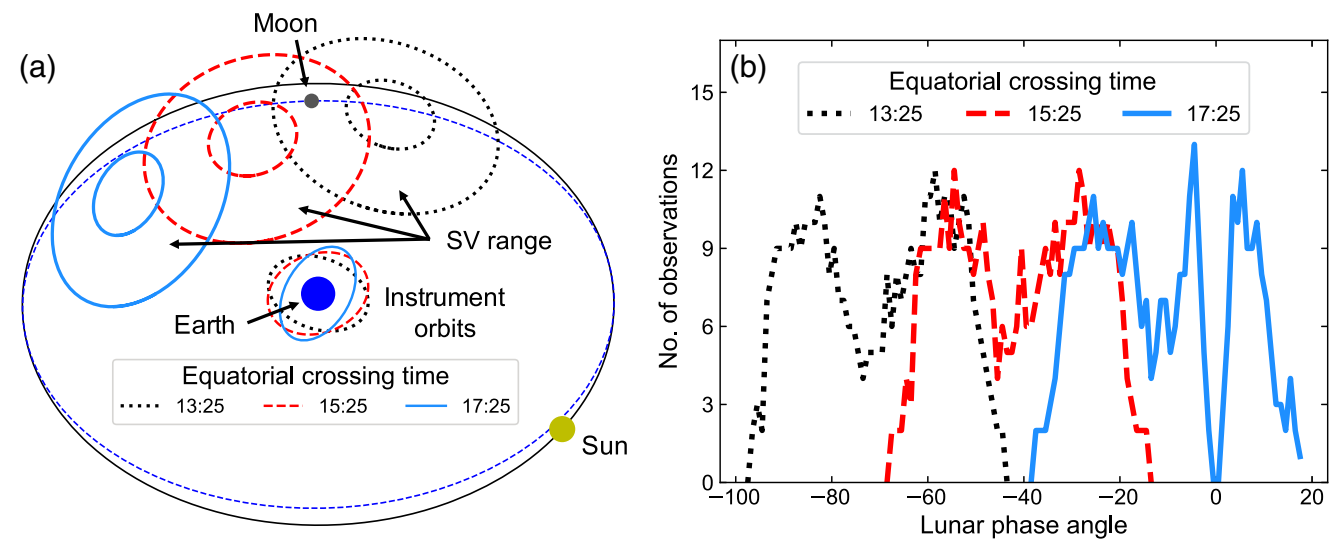

Fig. 8 (a) A diagram of the orbital geometries and the possible SV FOV ranges for VIIRS instruments at different equatorial crossing times (not to scale). The three sets of circles show the range of possible FOVs (using the same color and line style as the orbits) from the nominal pointing direction of the SV port (outer circle) to the pointing direction of the SV port at the maximum roll angle (inner circle), which we set to -15 deg here. (b) Histograms of the number of observation opportunities at a given lunar phase angle in 1-deg bins for each orbit in (a). These histograms show simulated data from 2017.

FOV ranges for parts of the orbit, which would indicate views at the same lunar phase for at least some observations are possible. The histograms in Fig. 8(b) confirm that this is the case and show significant overlap in the observations in the $-60 \mathrm{deg}$ to -50 deg range for the 13:25 and 15:25 orbits and -30 deg to -20 deg range for the 15:25 and 17:25 orbits. The 17:25 orbit histogram shows that at 0 -deg phase, there are no observations. Since the Moon's orbit is tilted by $\sim 5$ deg relative to the ecliptic, the nearly perfect alignment between the Sun, Moon, and instrument that is required for observation phase angles around 0 deg is rare and may not produce any observations within the allowed parameter range.

Lunar roll maneuvers provide an often used calibration data set for many Earth-observing instruments. The simulations and predictions shown here are just a small subset of the number of possible configurations that we could analyze. However, they do provide information on what to expect for different instrument and orbital configurations and help to determine the optimum lunar phase angle that should be chosen for the maneuvers.

\section{Lunar Observations from Pitch Maneuvers}

For MODIS and VIIRS, pitch maneuvers (about the $y$ axis of the ICS) are used in order to characterize the RVS of the TEB when looking at deep space through the EV port during the maneuver. ${ }^{20-22}$ For some of these maneuvers, the timing is chosen such that the Moon is visible near the nadir AOI during the maneuver so that an extra observation of the Moon takes place away from the nominal phase angles mentioned previously. During these nadir observations, multiple instruments on board the same platform can view the Moon simultaneously, which provides an opportunity for instrument intercomparison. We can use the tool developed here in order to predict the orbit that is ideal for operational purposes, however, we have to make a few changes to the geometric restrictions to suit the pitch maneuver.

Since the EV port has a wide FOV, unlike the SV port where the observations from roll maneuvers occur, pitch maneuvers provide a large number of possible lunar observations over wide phase angle and observation angle ranges. However, if we set restrictions on our observations based on the same parameters as the roll maneuver, the instrument could potentially be at an arbitrary point in the orbit when the observation criteria are met, which could put the satellite on the opposite side of the Earth or in a position where a pitch maneuver could compromise instrument safety. We find it is best to first set a restriction on the location of the satellite in its orbit to ensure that it is well within the night-time portion of the orbit. Based on previous pitch maneuvers, the lunar observation is typically set to be near the end of the first quarter of the night 
time portion of the orbit, which means the phase angle for the Sun-Earth-instrument system $\alpha_{\text {SEI }}$ is $\sim 135$ deg (see Fig. 4). So, instead using the restrictions of Eq. (2), we can use the following:

$$
\begin{aligned}
& \alpha_{\mathrm{SEI}} \approx 135 \mathrm{deg} \\
& V_{\text {lat,min }} \leq T_{\text {lat }} \leq V_{\text {lat,max }} .
\end{aligned}
$$

Here we have added a view latitude $\mathrm{min} / \mathrm{max}$ parameters to allow us to set a target range for the view latitude in the OCS, which will set the angle from nadir where the lunar observation will take place ( $V_{\text {lat }}=0$ is at nadir). Since the pitch maneuver will rotate completely around back to the original satellite position, the rotation angle restriction has been removed. Also the lunar phase angle restriction has been removed since the view angle through the EV port will narrow down the observable phase angles as will be shown in Sec. 4.1. However, in principle, this criteria can be added if desired.

For Terra, three pitch maneuvers have been performed on orbit, two of which viewed the Moon (April 14, 2003 and August 5, 2017), as seen in Table 2. For each of the Terra pitch maneuvers, the observations were chosen such that the Moon was near the nadir view angle in the EV port, which means that the magnitude of the lunar phase angle is going to be near that of the instrument $\beta$ angle (the angle that the solar vector makes with the instrument orbital plane). The value of $\alpha_{\mathrm{SEI}}$ in each case is near the 135-deg target mentioned above, with slight offsets owing to the actual timing of the pitch maneuver and pitch rate. SNPP and N20 have each performed one pitch maneuver on orbit; however, both were performed at lunar phase angles near a new Moon, so the Moon was not observed. To date, Aqua has not performed a pitch maneuver. A diagram of a pitch maneuver for Terra can be seen in Fig. 9(a), with the corresponding lunar images from the 2003 event shown in Fig. 9(b).

Table 2 Parameters for the two Terra MODIS lunar pitch maneuvers. The parameters are calculated at the center time of the observation. The $\beta$ angle is the angle of the solar vector relative to the instrument orbital plane. The nadir angle is the angular offset of the Moon from the EV port nadir vector.

\begin{tabular}{lccccc}
\hline \hline Observation time (UTC) & Phase (deg) & $\beta$ (deg) & Pitch (deg) & $\alpha_{\text {SEI }}(\mathrm{deg})$ & Nadir angle (deg) \\
\hline 2003/04/14 to 22:09:30 & -27.7 & 20.5 & 113.6 & 129.8 & 0.3 \\
2017/08/05 to 23:29:54 & -20.4 & 20.0 & 138.5 & 137.3 & 0.2 \\
\hline \hline
\end{tabular}

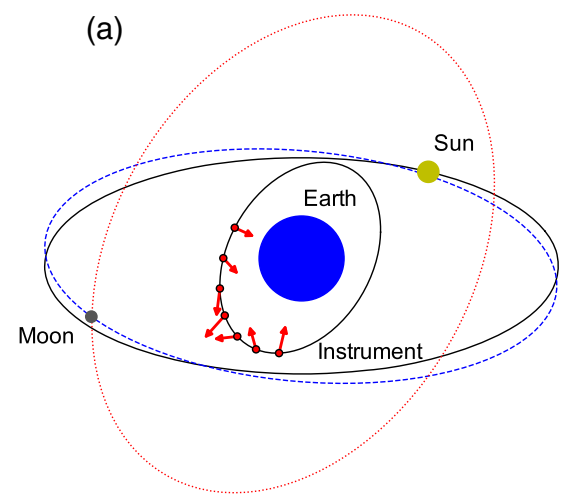

(b)
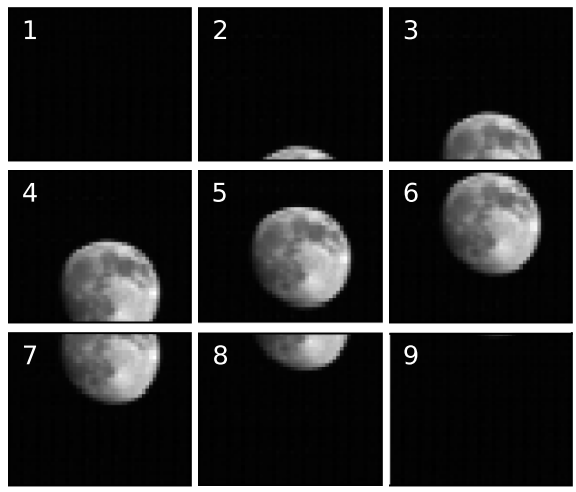

Fig. 9 Terra MODIS pitch maneuver on April 14, 2003. (a) A diagram of the maneuver showing the relative locations of the Sun, Moon, Earth, and the Terra satellite (not to scale). The blue dashed line shows the orbital plane of the Moon and the solid black line shows the ecliptic. The arrows show the pointing direction of the center of the EV port during the maneuver, which traces out a view path in the instrument orbital plane, indicated by the red dotted line. The maneuver begins when the satellite is in the lower portion of this diagram. (b) A sequence of images for Terra MODIS band 1 during the pitch maneuver. The time sequence of the images goes from left to right and then from top to bottom, as numbered. 


\subsection{Pitch Maneuver Orbit Simulations}

Since pitch maneuvers provide valuable information regarding the on-orbit changes to the TEB RVS as well as additional lunar observation opportunities, ${ }^{20,21,22}$ it is important for each mission to understand the future opportunities that will be available. The relative positions of the Sun, Earth, and Moon can be predicted with high accuracy for many decades in the future, ${ }^{29}$ but the actual instrument positions cannot be projected long term since various maneuvers are performed throughout each mission in order to provide small corrections to the orbital drift. However, we can simulate the orbits of these instruments for an arbitrary time period using the current orbital parameters (mean motion, eccentricity, etc.) and a specified equatorial crossing time.

Heading into the next decade, the MODIS instruments will reach 20 years of on-orbit operation (2020 for Terra and 2022 for Aqua) and VIIRS will reach 10 (2021 for SNPP and 2027 for N20). For VIIRS, there is also the expected launch of JPSS-2 and JPSS-3 in 2022 and 2026, respectively. For each of these instruments, it will be desirable to perform a pitch maneuver for instrument characterization near these milestones or shortly after the launch of the new instruments in order to monitor the instrument performance. Using simulated orbital data, we can predict the approximate timing of such observations.

For the MODIS instruments, there are late mission plans under development which will alter the orbits of the satellites from the current configuration. For Terra, the last orbital adjustment maneuvers will likely occur within the next few years, after which the orbit will be allowed to drift to earlier equatorial crossing times. For Aqua, there are plans to lower its orbit early in the next decade and continue obtaining science data for as long as the instruments are operational. To understand how these changes will affect the lunar observations during pitch maneuvers, we can simulate additional orbits at other equatorial crossing times for Terra MODIS, similar to what was done for SNPP in Sec. 3.1.

For pitch maneuvers, the observations occur through the EV port instead of the SV port, which has a much wider FOV of $\pm 55 \mathrm{deg}$ from the EV nadir. This means that there are a large number of potential observations over successive orbits until the Moon is no longer in this FOV. Also since pitch maneuvers use a full rotation back to the original spacecraft orientation, observations will be possible during every lunar cycle over a range of phase angles. For this work, we will analyze a set of simulated orbits for the year 2020, which includes instruments in the Terra MODIS configuration with descending daytime equatorial crossing times (local) of 09:30, 10:00, and 10:30, Aqua MODIS with an ascending daytime equatorial crossing time of 13:35, and SNPP VIIRS with an ascending daytime equatorial crossing time of 13:25. For N20 VIIRS, the same orbit from SNPP can be assumed for the purposes of this simulation. For each of these configurations, we calculated all of the predicted pitch maneuver observations using the observation conditions from Eq. (8) over the full EV FOV for analysis. In Fig. 10, we show the lunar phase angle when the observation is near nadir in each lunar cycle for each of the orbits listed above. In each case, there is an $\sim 12$ - deg change in the lunar phase angle at nadir over the course of the year, which corresponds to changes in the satellite orbit $\beta$ angle and the relative orbit of the Moon. We can also note that an equatorial crossing time change results in a

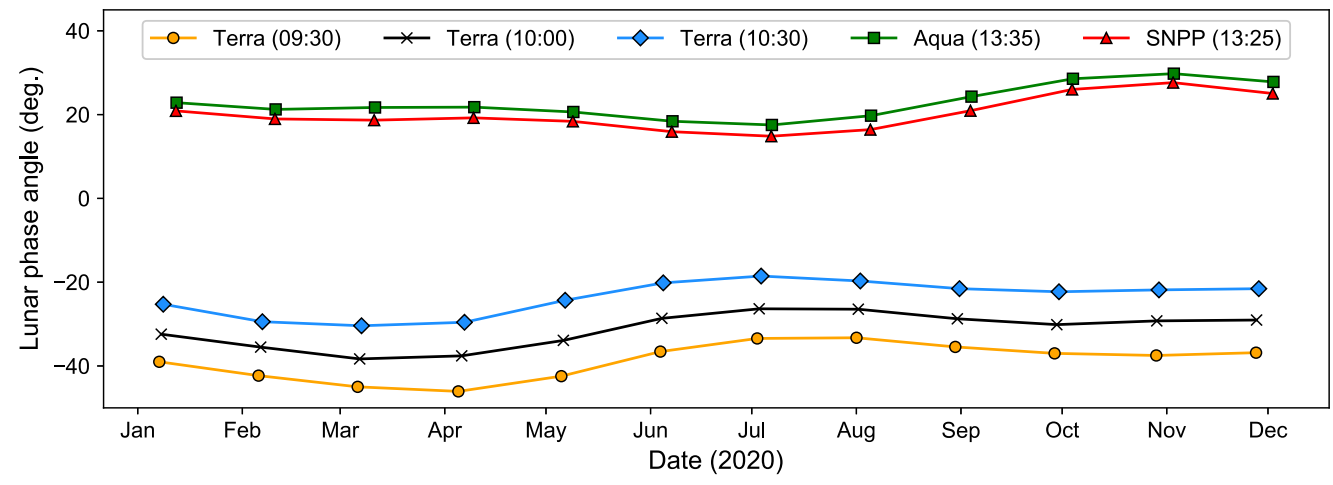

Fig. 10 A plot of the predicted lunar phase angle $\alpha_{\mathrm{SMI}}$ for pitch maneuver observations at the EV nadir for each lunar cycle for different instrument orbits. 


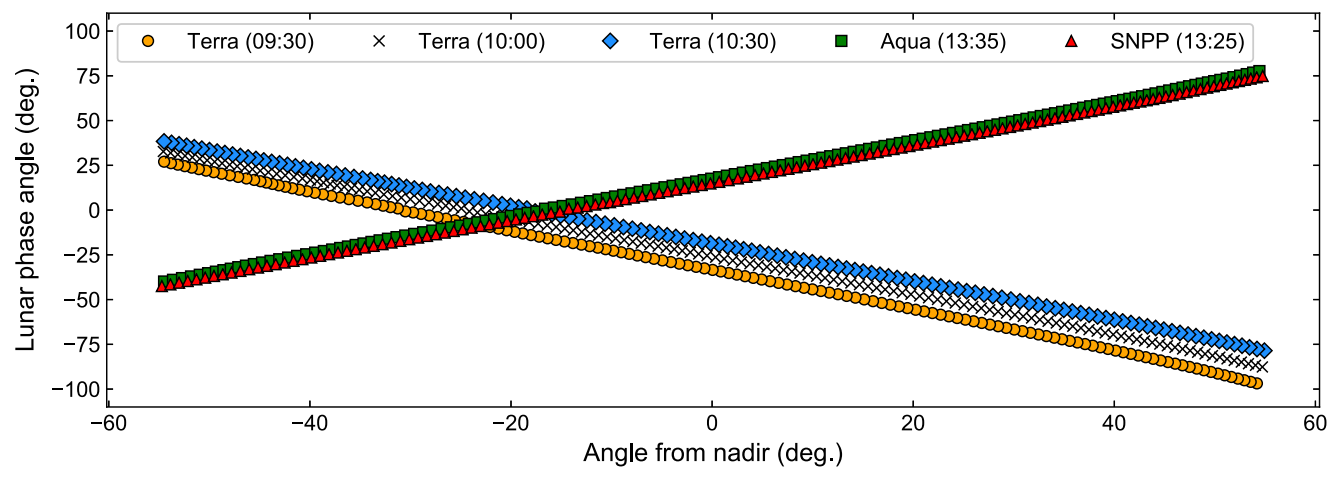

Fig. 11 Comparison of the observation angle from the EV nadir versus the observed lunar phase angle $\alpha_{\text {SMI }}$. The data in this plot are from the late June / early July 2020 lunar cycle for Terra MODIS at three different equatorial crossing times (daytime, descending) and for Aqua MODIS and SNPP VIIRS (daytime, ascending). N20 is not shown because both SNPP and N20 are at opposite points in the same orbit.

nearly constant shift in the phase angle between each of the orbital configurations. Since the instruments are in low-Earth orbit and the observations discussed here occur in the instrument orbital plane, there is a direct correlation between the equatorial crossing time and the observed phase angle. These changes are at $\sim 15 \mathrm{deg}$ per h change in the crossing time, as expected, since a full 24-h change in the crossing time will yield the same orbit with a 360-deg phase change.

In Fig. 11, we show the observation angle from the EV nadir versus the observed lunar phase angle for a single lunar cycle in 2020 from late June / early July. The observation angle is defined as being negative at the beginning of the scan and positive at the end of the scan. Here we can see that the observation angle and phase angle have a nearly linear relationship, with changes in each that are on the order of $1 \mathrm{deg}$ per orbit. For each lunar cycle, the Moon can be observed for consecutive orbits for around 10 days over a lunar phase angle range of $\sim 120 \mathrm{deg}$. Since the beginning of the lunar cycle is at a negative phase angle, the Terra observations start at a positive observation angle and the Aqua and SNPP observations start at a negative observation angle. For Terra, this analysis shows that for drifts in the orbit to earlier equatorial crossing times, the observed lunar phase angles shift downward as seen previously in Fig. 10. We also see a small shift in the observations associated with the slight difference in equatorial crossing time between Aqua and SNPP.

Since the orbits of Aqua and SNPP are opposite that of Terra, they do not observe the Moon at the same phase angle when at nadir, but they do have a crossing point near a full moon at $\sim 18 \mathrm{deg}$ away from nadir. However, these plots indicate there are a wide range of potential phase angles that could be used for near simultaneous observations if the AOI is allowed to differ. We can also note that the nominal phase angle ranges listed in Table 1 are not observable during the pitch maneuvers owing to the $\beta$-angle offset of the orbits.

We can also make more specific predictions for potential pitch observations as seen in Table 3. Here we list the predictions for both near-term pitch observations for several instrument configurations as well as some long-term predictions associated with various instrument milestone events as discussed previously. In this table, we show the expected lunar phase angle at three different observation angles centered around the date of the near-nadir observation. Although the precise timing of the observation cannot be predicted this far in advance, the dates of the observations will be largely driven by the location of the Moon and the equatorial crossing time.

For Terra MODIS, we show predictions for the years 2020 and 2025, which represents 20 and 25 years of on-orbit operation. Since Terra is expected to drift in its orbit in the next decade, we show predictions for three different equatorial crossing times. In each case, the lunar phase angle changes by approximately the same amount as the observation angle. Also the angle at nadir (0 deg) varies with the season as shown in Fig. 10. Since Aqua has not yet performed a pitch maneuver, we show two candidate observations in early July 2020 and late June 2021. In each 
Table 3 Future pitch maneuver opportunities for the MODIS and VIIRS instruments. The lunar phase angles are shown at view angles of $-15 \mathrm{deg}, 0 \mathrm{deg}$, and $15 \mathrm{deg}$ away from the EV nadir directions. The observation dates are listed for the 0-deg view. The three observations can span over multiple days.

\begin{tabular}{|c|c|c|c|c|c|}
\hline \multirow[b]{2}{*}{ Instrument } & \multirow[b]{2}{*}{ Orbit type } & \multirow[b]{2}{*}{ Observation date } & \multicolumn{3}{|c|}{ Lunar phase at angle (deg) } \\
\hline & & & -15 & 0 & +15 \\
\hline \multirow[t]{6}{*}{ Terra MODIS } & 09:30 descending & $2020 / 07 / 02$ & -18 & -33 & -50 \\
\hline & 10:00 descending & $2020 / 07 / 03$ & -10 & -26 & -42 \\
\hline & $10: 30$ descending & $2020 / 07 / 03$ & -3 & -19 & -34 \\
\hline & 09:30 descending & $2025 / 01 / 10$ & -26 & -40 & -55 \\
\hline & 10:00 descending & $2025 / 01 / 11$ & -19 & -33 & -48 \\
\hline & $10: 30$ descending & $2025 / 01 / 11$ & -13 & -27 & -42 \\
\hline \multirow[t]{2}{*}{ Aqua MODIS } & 13:35 ascending & $2020 / 07 / 06$ & 2 & 18 & 33 \\
\hline & $13: 35$ ascending & $2021 / 06 / 26$ & 3 & 18 & 33 \\
\hline \multirow[t]{4}{*}{ SNPP/N20 VIIRS } & $13: 25$ ascending & $2020 / 07 / 06$ & -1 & 15 & 31 \\
\hline & $13: 25$ ascending & $2021 / 10 / 23$ & 12 & 28 & 45 \\
\hline & $13: 25$ ascending & 2023/01/08 & 8 & 22 & 36 \\
\hline & 13:25 ascending & $2027 / 11 / 16$ & 14 & 29 & 43 \\
\hline
\end{tabular}

case, the observation phase angles are approximately the same because they occur at nearly the same time of year.

For VIIRS, since SNPP and N20 are at opposite ends of the same orbit, we show the candidate observations together. Additionally, future JPSS launches will also be in an orbit at a similar equatorial crossing time, so the observations predicted after those launches would also be candidates for those instruments. Here we show a prediction for 2020 in order to compare to the MODIS predictions. We also show predictions for 2021 (10 years after SNPP launch), 2023 (after the launch of JPSS-2), and 2027 (10 years after the N20 launch, after the launch of JPSS-3).

Pitch maneuvers occur rarely throughout the MODIS and VIIRS missions, but they do provide valuable information on the TEB RVS and additional lunar calibration opportunities. Our analysis shows that lunar observations will be available during a pitch maneuver during every lunar cycle for $>100$ successive orbits. This provides a large number of options to choose from depending on the type of comparison that is desired.

\section{Planet and Star Observations}

The tool developed in this work can be used for general observation planning as well as for scheduling lunar observations as discussed above with just a few minor changes. In particular, it can be used for determining the timing of observations through the SV port of the bright outer planets (Mars, Jupiter, and Saturn) for high-gain bands in MODIS and VIIRS as well as the observations of stars in the high-gain stage of the VIIRS DNB. ${ }^{7,15}$ For this purpose, the tool can be easily modified to use the specified planet or star as the target. Since there is no need to apply a phase angle restriction in this case, the only geometric requirements are specified in Eq. (2). In this case, the values of $\rho_{\min }$ and $\rho_{\max }$ specify the angular FOV of the SV port, which is $4.1 \mathrm{deg}$ for MODIS and 0.9-deg VIIRS in total. The angular FOV for VIIRS is lower because it does not use subpixel aggregation in the SV port for the imaging (I, 375-m nadir resolution) and moderate resolution (M, 750-m nadir resolution) bands, but still limits the 

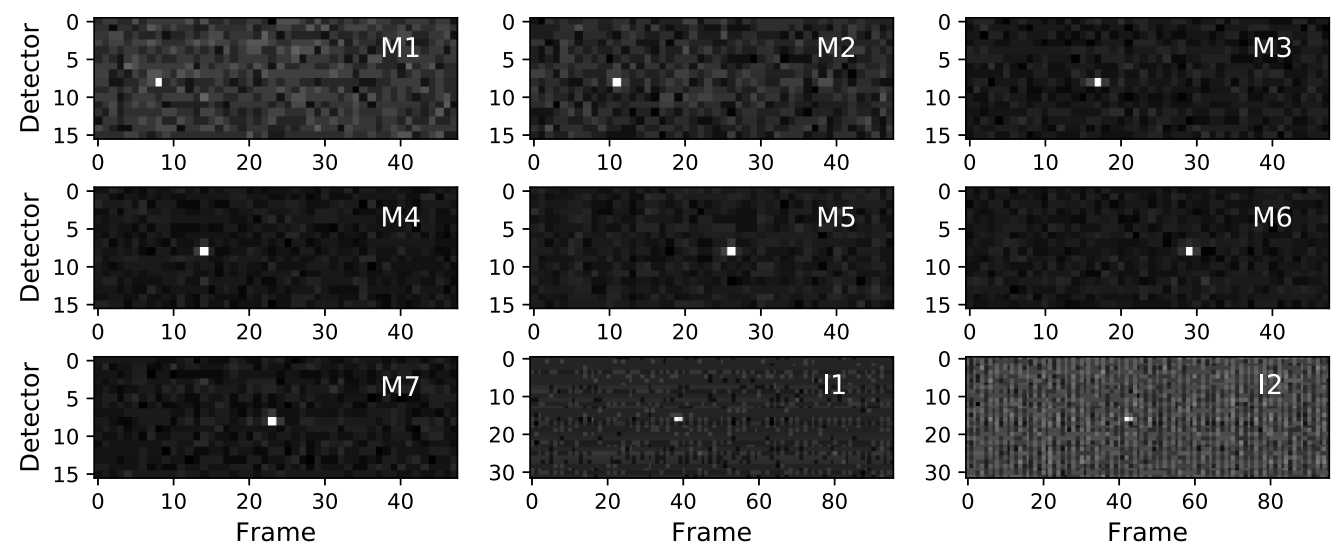

Fig. 12 SNPP VIIRS observations of Jupiter for the visible and near-infrared bands from January 24, 2012. Each band image comes from the same scan.

number of SV frames to 48 for the M-bands and 96 for the I-bands in each scan. For most bands on MODIS, the gain is too low to observe stars or planets, and only the high-gain ocean color bands 13 to 16 are sensitive enough for the bright planets. For VIIRS, the visible and near-infrared bands, M1 to M7, I1, and I2 can observe the bright planets. For the VIIRS DNB, the highgain stage saturates when viewing the planets, but the mid-gain stage has observations below the saturation level.

Although the planetary observations are at low-signal level, they still might be used for other sensor performance assessments, such as a spatial registration characterization between the bands. An example of these planetary observations can be seen in Fig. 12 for Jupiter in SNPP VIIRS. For each band, the image is taken from the same scan. Here we see that Jupiter appears horizontally offset in the images because of the relative location of each band on the focal plane assembly. Since planets will appear as near point sources for these instruments, with enough observations, we should be able to track changes in the spatial registration in both the scan and track directions.

For the VIIRS DNB, there has been some previous work which uses bright stars to compute the on-orbit gain change of the high-gain stage. ${ }^{7}$ Since the DNB has a broad spectral response over the visible range, stellar observations can be useful for determining the spectral response change on orbit by tracking the gain change as measured by stars of a different spectral class. This is of particular importance for SNPP, which experienced a change in the relative spectral response owing to contamination on the rotating telescope assembly mirror early in the mission. ${ }^{30}$ For the given orientation of the SV port on the VIIRS instrument in its orbit, $\sim 3500$ bright stars from the Yale Bright Start Catalog are found to have potential observations. ${ }^{15}$ Since there are so many stars that can be viewed by the DNB across all the different spectral classes and visual magnitudes, proper identification of the observations is essential for making use of this data.

The planet and star observations provide only low-signal data sets, however, they occur without using additional mission resources since no spacecraft maneuvering is required. Using this planning tool, we can go through the entire data archive for a given mission in order to extract a large amount of useful data for sensor performance assessments.

\section{Conclusion}

Calculating the maneuver parameters for lunar observations is critical for on-orbit calibrations of Earth-observing instruments that use the Moon. In this work, we developed a tool based on the SPICE toolkit which can accurately predict lunar, planetary, and stellar observations in a variety of instrument and orbital configurations. This tool is currently in use by the MODIS and VIIRS Characterization Support Teams in order to schedule the lunar calibration activities that occur on a near monthly basis. We tested the tool versus the historical lunar observations from roll maneuvers for the MODIS and VIIRS instruments and found that the predicted observation times match 
the measured times to within a single scan for each instrument. The accuracy of the tool when analyzing these past events gives us confidence in the predictions for the simulated orbits and future observation predictions discussed in the text. We then analyzed the changes in the possible observations from roll maneuvers using different observation ports and at different equatorial crossing times using simulated orbital data based on the orbit of SNPP as an example. We found that the MODIS and VIIRS SV pointing configurations produce similar observation opportunities at opposite points in the satellite orbits. We also showed that changes in the equatorial crossing time shifted the observation opportunities by $\sim 15 \mathrm{deg}$ per $\mathrm{h}$, as expected.

The tool was also used to calculate the parameters for lunar observations from pitch maneuvers, which uses the EV port instead of the SV port for the observations. This required minor changes in the geometric restrictions used to find the observation times. Using simulated orbital data, we generated predictions of pitch observations for future milestone dates for the MODIS and VIIRS missions. We also analyzed the relationship of the lunar phase angle during the pitch observation with the angular offset in the EV port and as a function of the satellite $\beta$ angle, which varies annually. We found that there are a large number of potential pitch observation opportunities available during every lunar cycle. Also for instruments in different orbits, observations at the nearly same lunar phase angle are possible.

We also extended the tool to find the timing of planetary observations for MODIS and VIIRS and for stellar observations for the VIIRS DNB. These observations occur in the SV port and do not require spacecraft maneuvers. The tool can be used in order to find the proper datasets in the data archives of a given mission. For VIIRS in particular, the planetary observations can be useful for a spatial registration characterization, since the planet is a point-like source observed across many of the visible/near-infrared bands. The stellar observations have already been used previously for calibration of the high-gain stage of the VIIRS DNB, and this tool could help in the identification of the observed stars, which will allow us to obtain the correct spectral data.

The methods used in these tools can also be extended to other observation types as well, such as planning SD calibration events and for finding the timing of EV overpasses of selected sites at specified angles. These tools are already in use by the MODIS and VIIRS Characterization Support Teams and may be the subject of future work.

\section{Acknowledgments}

The work presented here would not be possible without the dedicated efforts of the members of the MODIS and VIIRS Characterization Support Teams, past and present, who have a wealth of knowledge in the area of satellite remote sensing. In particular, we would like to thank Amit Angal of SSAI and MCST for his helpful comments and discussions about this work. We would also like to thank Daniel Todaro and Michael Lucci of SSAI and the MODIS Instrument Operations Team for helpful discussions related to the instrument orbital simulations. Finally, we would like to thank the reviewers for their helpful comments and suggestions for improvements to the manuscript. The authors declare no conflicts of interest.

\section{References}

1. X. Xiong et al., "MODIS on-orbit calibration and characterization," Metrologia 40, S89-S92 (2003).

2. X. Xiong et al., "VIIRS on-orbit calibration methodologies and performance," J. Geophys. Res. 119(9), 5065-5078 (2014).

3. J. Sun et al., "MODIS reflective solar bands on-orbit lunar calibration," IEEE Trans. Geosci. Remote Sens. 45(7), 2383-2393 (2007).

4. X. Xiong et al., "Lunar calibration and performance for S-NPP VIIRS reflective solar bands," IEEE Trans. Geosci. Remote Sens. 54(2), 1052-1061 (2015).

5. J. Sun et al., "Time-dependent response versus scan angle for MODIS reflective solar bands," IEEE Trans. Geosci. Remote Sens. 52(6), 3159-3174 (2014).

6. B. N. Wenny et al., "Evaluation of Terra and Aqua MODIS thermal emissive band response versus scan angle," Proc. SPIE 9218, 92181L (2014). 
7. J. Fulbright and X. Xiong, "Suomi-NPP VIIRS day/night band calibration with stars," Proc. SPIE 9607, 96071S (2015).

8. H. H. Kieffer and T. C. Stone, "The spectral irradiance of the Moon," Astron. J. 129(6), 2887-2901 (1999).

9. R. A. Barnes et al., "Changes in the radiometric stability of SeaWiFS determined from lunar and solar measurements," Appl. Opt. 38(21), 4649-4664 (1997).

10. C. J. Bruegge et al., "Validating the MISR radiometric scale for the ocean aerosol science community," in Post-Launch Calibration of Satellite Sensors, S. A. Morain and A. M. Budge, CRC Press, Boca Raton, Florida, pp. 103-116 (2003).

11. B. Markham et al., "Landsat- 8 operational land imager radiometric calibration and stability," Remote Sens. 6(12), 12275-12308 (2014).

12. T. Wilson, A. Angal, and X. Xiong, "Sensor performance assessment for Terra and Aqua modis using unscheduled lunar observations," Proc. SPIE 10785, 1078519 (2018).

13. J. Sun and X. Xiong, "Solar and lunar observation planning for Earth-observing sensor," Proc. SPIE 8176, 817610 (2011).

14. J. Butler et al., "An overview of Suomi NPP VIIRS calibration maneuvers," Proc. SPIE 8510, 85101J (2012).

15. T. Wilson and X. Xiong, "Scheduling observations of celestial objects for Earth observing sensor calibration," Proc. SPIE 10000, 1000011 (2016).

16. Y. Yamaguchi et al., "Overview of advanced spaceborne thermal emission reflection radiometer (ASTER)," IEEE Trans. Geosci. Remote Sens. 36(4), 1062-1071 (1998).

17. C. H. Acton, "Ancillary data services of NASA's Navigation and Ancillary Information Facility," Planet. Space Sci. 44, 65-70 (1996).

18. S. Hughes et al., "Verification and validation of the general mission analysis tool (GMAT)," in AIAA/AAS Astrodyn. Spec. Conf., p. 4151 (2014).

19. ATBD, VIIRS GEO, "Joint polar satellite system (JPSS) visible infrared imaging radiometer suite (VIIRS) sensor data record (SDR) geolocation algorithm theoretical basis document (ATBD)," (2013).

20. X. Xiong et al., "On-orbit monitoring of MODIS thermal emissive bands response versus scan angle," Proc. SPIE 6744, 67441I (2007).

21. A. Wu et al., "Assessment of the NPP VIIRS RVS for the thermal emissive bands using the first pitch maneuver observations," Proc. SPIE 8510, 85101Q (2012).

22. X. Xiong et al., "MODIS and VIIRS on-orbit calibration and characterization using observations from spacecraft pitch maneuvers," Proc. SPIE 10764, 107640V (2018).

23. V. V. Salomonson et al., "An overview of Earth Observing System MODIS instrument and associated data systems performance," in Proc. Int. Geosci. Remote Sens. Symp., vol. 2, pp. 1174-1176 (2002).

24. X. Xiong et al., "Updates of moderate resolution imaging spectroradiometer on-orbit calibration uncertainty assessments," J. Appl. Remote Sens. 12(3), 034001 (2018).

25. N. Lei et al., "SNPP VIIRS RSB earth view reflectance uncertainty," in IEEE Int. Geosci. Remote Sens. Symp. (IGARSS), pp. 5916-5919 (2017).

26. J. Sun, X. Xiong, and W. L. Barnes, "MODIS reflective solar bands unscheduled lunar observations," Proc. SPIE 6677, 66771K (2007).

27. Z. Wang, X. Xiong, and J. P. Fulbright, "Suomi-NPP VIIRS unscheduled lunar observations," Proc. SPIE 10000, 1000012 (2016).

28. T. Wilson et al., "Development and implementation of an electronic crosstalk correction for bands 27-30 in Terra MODIS collection 6," Remote Sens. 9(6), 569 (2017).

29. W. Folkner, J. Williams, and D. Boggs, "The planetary and lunar ephemeris DE 421," IPN Progr. Rep. 42(178), 1-34 (2009).

30. N. Lei, X. Xiong, and B. Guenther, "Modeling the detector radiometric gains of the Suomi NPP VIIRS reflective solar bands," IEEE Trans. Geosci. Remote Sens. 53(3), 1565-1573 (2015).

Truman Wilson received his $\mathrm{BS}$ and $\mathrm{PhD}$ degrees in physics from Colorado State University, Fort Collins, Colorado, USA. After completing his PhD, he did postdoctoral work at the National Institute of Standards and Technology, Gaithersburg, Maryland, USA. Currently, he is a senior 
scientific researcher at the Science Systems and Applications, Inc., Lanham, Maryland, USA, where he is a member of both the MODIS and VIIRS Characterization Support Teams.

Xiaoxiong Xiong is an optical physicist at NASA Goddard Space Flight Center. He currently serves as the MODIS project scientist, the VIIRS calibration scientist, and the technical lead for the MODIS/VIIRS Characterization Support Team (MCST/VCST). He is also a member of the CLARREO Science Team and CLARREO Pathfinder Team. Before joining the NASA/GSFC, he also worked as a guest researcher at the National Institute of Standards and Technology (NIST) on optical instrumentation and nonlinear optics. 